\title{
One-dimensional cluster array at the three-phase contact line in diluted colloids subjected to ac electric fields
}

\author{
M. Pichumani, M. Giuliani, ${ }^{*}$ and W. González-Viñas ${ }^{\dagger}$ \\ Department of Physics and Applied Mathematics, University of Navarra, Irunlarrea s/n, E-31080 Pamplona, Spain
}

(Received 3 December 2010; published 26 April 2011)

\begin{abstract}
Colloidal particles provide an efficient means of building multiple scale structured materials from colloidal dispersions. In this Brief Report we account for experimental evidence on the formation of a colloidal cluster array at a three-phase contact line. We study the influence of low frequency external alternating electric fields on a diluted colloidal dispersion opened to the air. We focus on the cluster formation and their evolution in the meniscus by measuring characteristic times and lengths. We observe that the clusters are separated by a well-defined length and that, in our experimental conditions, they survive between 5 and 15 min. These new results could be of technological relevance in building tailored colloidal structures in nonpatterned substrates.
\end{abstract}

DOI: 10.1103/PhysRevE.83.047301

PACS number(s): 47.57.J-, 81.16.Rf

Nonequilibrium transitions by external forces in colloidal systems have gained attention among research groups [1-7] lately. Normally, these kind of transitions are more complex in nature because their interrelated parameters are affected by the external forces. These transitions occur when the state of the system becomes unstable, as the control parameters are varied and usually lead to pattern formation [8,9]. Flow dynamics plays an important role in the evolution of the system by transporting colloidal particles in the transitions [10]. The dynamic response of the colloidal dispersion to the applied external fields describes the basic functionality of the system and their inter-relative behavior (between clusters, and between clusters and substrates). Previous works [11-13] study the influence of external fields on bulk colloidal dispersions. Here we focus our attention in the meniscus of a colloid with a free surface rather than in the bulk dispersion. Due to minimal evaporation in our setup, the contact line does not recede significantly during the measurement time. Consequently, the cluster formation is not related to a fingering instability in the contact line [14-16]. Also, we explore the effect of weak external ac electric fields in this region. Upon comparison with the surface and other kinds of instabilities [17,18], the one which we obtain is mainly comprised of two phenomena: (a) electrokinetic and (b) electrowetting [19,20]. The possible effect from electrokinetic phenomena (mainly electrophoresis) on the dispersion is the formation of a concentration gradient (maintaining a high concentration of particles near the contact line which is greater than the initial concentration). On the other hand, ac electrowetting induces local flows in the meniscus which may form clusters. Both contributions set a threshold for the forcing in order to obtain clusters. In this work, we investigate the effect of external weak alternating electric fields on a diluted colloidal dispersion. Under these conditions, the interparticle interactions may play a critical role in transport properties [21-23]. We focus on a region of the parameter space where clusters form. We study three relevant aspects: (i) the response of the clusters to the applied field;

\footnotetext{
*Present address: Department of Physics, University of Guelph, Canada.

†wens@fisica.unav.es
}

(ii) the evolution of the system by measuring the time required for the cluster formation; and (iii) the one-dimensional spatial distribution of the clusters and their evolution in time. The aim of this work is to gain insight regarding the mechanisms involved in colloidal cluster formation and their evolution.

The experimental setup and the cell are shown in Fig. 1. The substrates used for all the experiments are of standard glass (of size $1.1 \times 17 \times 18 \mathrm{~mm}^{3}$ ) in which one of the sides $\left(17 \times 18 \mathrm{~mm}^{2}\right)$ is coated with a thin conductive layer of indium tin oxide (ITO). They are placed vertically in a Teflon cell (Fig. 1, right). The substrates are placed in such a way that the conductive side is in contact with the suspension. The thickness of the colloidal suspension between the two substrates is $1 \mathrm{~mm}$. To apply electric fields, we place two ceramic spacers (one side of each ceramic spacer is made conductive) between the substrates. Rubber O-rings are used to ensure that the cell along with the substrates and spacers are held tightly. All the experiments are performed at room temperature. We use a colloidal dispersion of spherical polystyrene particles of diameter $1.3 \mu \mathrm{m}$ (polydispersity is 0.039 ). These particles are suspended in water and their surface charge is $-7 \mu \mathrm{C} / \mathrm{cm}^{2}$. The dispersion was acquired from Dr. Paulke at Fraunhofer-IAP, Germany. We dilute this stock dispersion to $0.5 \%$ (w/w) concentration using ultrapure water.

The substrates are cleaned with nitrogen blow and they are mounted in the cell. A subset of the substrates was prepared with more hydrophilic character in order to evaluate the influence of the equilibrium contact angle on the cluster formation. Electric fields (ac) of square wave form are applied perpendicularly to the conducting substrates. We apply low voltage (of the order of $1 \mathrm{Vpp} / \mathrm{mm}$ ) and low frequency (of the order of $1 \mathrm{~Hz}$ ). We observe the region near the contact line using a microscope with an objective of magnification 10X. This microscope is attached to a complementary metal oxide semiconductor (CMOS) camera for capturing sequential frames (Fig. 2). The location of the contact line corresponds to the transition between the dark region (colloidal suspension) at the bottom and the white region (bare substrate) at the top. The area of observation corresponds to the central part of the contact line region with a field of view of $0.6 \mathrm{~mm}$. However, the observed phenomenology is common to the whole contact line (far from boundaries). 

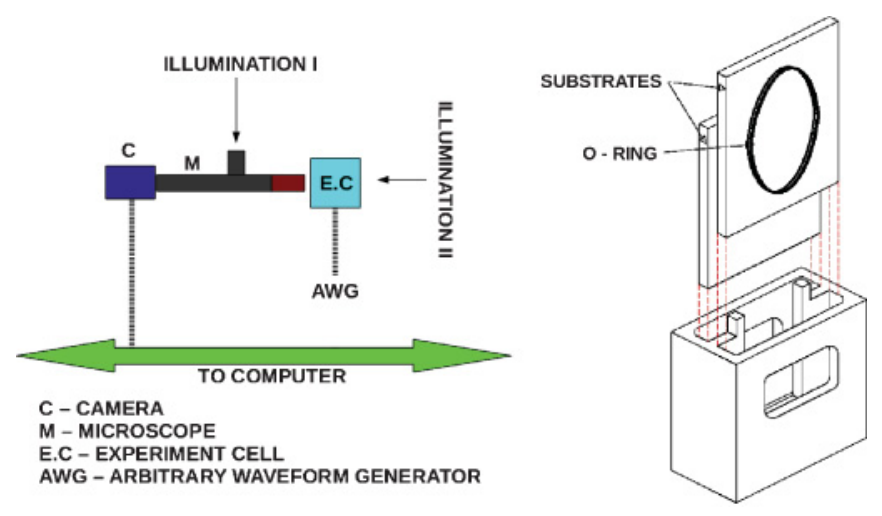

FIG. 1. (Color online) Sketch of the experimental setup (left) and of the cell (right).

After the dispersion is loaded into the cell (Fig. 2(a)), we apply the electric field and images of the system are saved sequentially. Due to the electrophoretic effect, the particles rhythmically get closer to the contact line. They are trapped in the meniscus and start to accumulate (Figs. 2(b) and 2(c)). After some time, the clusters start to initiate (Fig. 2(d)) and they continue to grow (Figs. 2(e) and 2(f)) until they stabilize their size. During the growing stage, clusters change their shape at the same frequency as the applied field. Finally, the clusters will age or dissociate (Fig. 2(g)). (See clip A in [24].)

In order to obtain an initial picture about the system, we observe the response of particles to the applied field. From the stored frames, we construct a space time diagram from which their frequency is obtained. We measured the response of the particles to the external forcing in two cases: (i) by keeping the frequency constant $(1 \mathrm{~Hz})$ and varying the amplitude and (ii) by keeping the amplitude constant (1.8 Vpp) and varying the frequency. We checked that for the range of applied amplitudes (from 1.8 to $2.4 \mathrm{Vpp}$ ) at a constant frequency of $1 \mathrm{~Hz}$, the measured frequencies of the particles coincide with

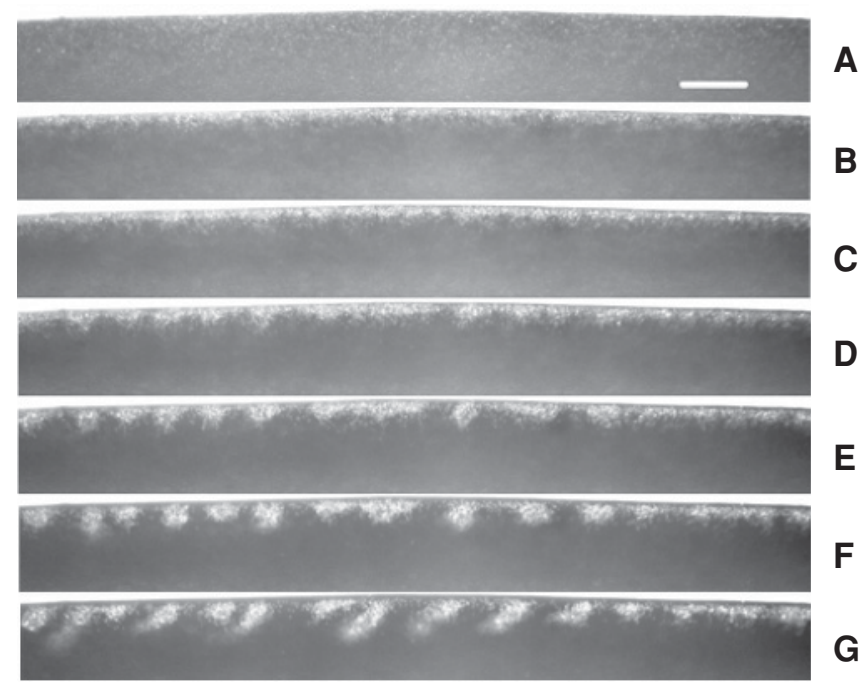

FIG. 2. Snapshots of the contact line region (front view) at $1.8 \mathrm{Vpp}$ and $0.8 \mathrm{~Hz}$. The bright regions are particles or clusters. The frames are captured at intervals of $1 \mathrm{~min}$ and the scale bar is $50 \mu \mathrm{m}$. The stages (A-G) are explained in the text.

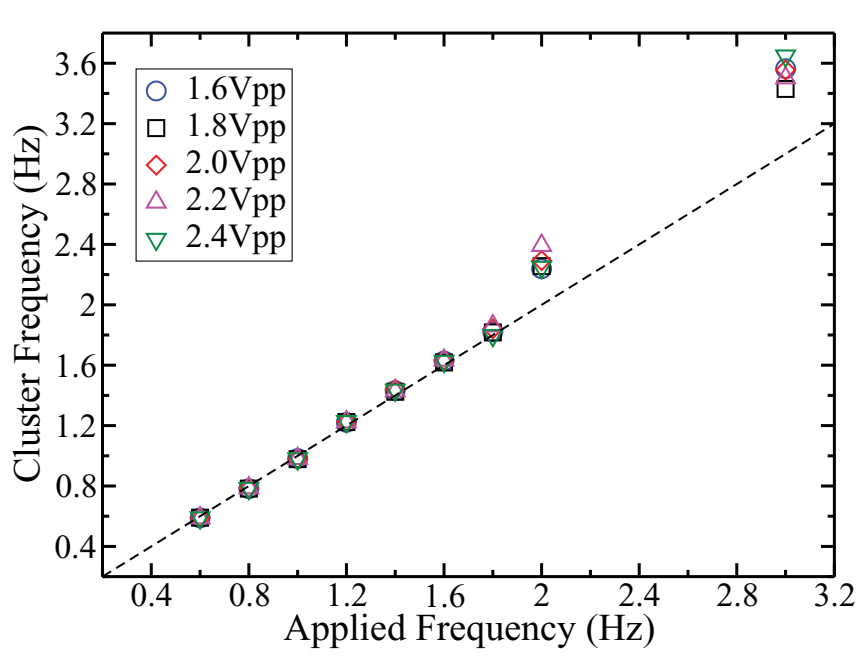

FIG. 3. (Color online) Frequency response of the clusters to the applied field. The dashed line is indicative of the applied frequencies.

the applied one $(1.00 \pm 0.06 \mathrm{~Hz})$. In both cases (i) and (ii), we confirm that in our experimental conditions, the particles follow the applied field adiabatically.

Concerning the response of clusters to the applied field, we define a horizontal line in the cluster region for all the experimental frames. Along this line the brightness is captured in such a way that the intensity varies when the clusters move and/or they change their intrinsic brightness. We construct the corresponding space-time diagram followed by a twodimensional fast Fourier transformation (FFT) which gives a frequency related to the clusters (Fig. 3). In the case of applied low frequencies $(0.6-1.8 \mathrm{~Hz})$, the clusters follow the forcing condition whereas the response is of higher values at applied high frequencies $(2.0-3.0 \mathrm{~Hz})$. To study further the behavior of clusters and their relevant parameters (in correlating their formation with the applied field), we measured the temporal scale at which they form or evolve. To estimate this, we measured the threshold time $\left(t_{c}\right)$ which we define as the time beyond which the clusters start to age or dissociate. The nondimensional threshold time $\left(f t_{c}\right)$ as a function of applied frequencies $(f)$ is shown in Fig. 4 and it increases proportionally to the increasing applied frequencies. In our experimental conditions, we did not observe any dependence of $f t_{c}$ with the strength of the applied field (data not shown).

The clusters are spatially distributed and they are separated by a length $\lambda$. We measured the characteristic length at the threshold time $t_{c}$ (Fig. 5). The characteristic length $\bar{\lambda}$ defines the mean spatial distance between the clusters calculated through the spatial auto-correlation function. If we consider for one applied amplitude (e.g., $2.4 \mathrm{Vpp}$ in Fig. 5), the characteristic length evolves as we increase the frequency. For one particular frequency (e.g., at $1.0 \mathrm{~Hz}$ ), a relative maximum in characteristic length is observed.

On applying the electric field, the electrophoretic effect supplies particles to the meniscus much more efficiently than the Brownian motion does. As it is stated above, surface forces trap particles in the meniscus. Secondly, ac electrowetting [19] generates a flow $[25,26]$ near the contact line (due to the increase and decrease in the contact angle) that may become unstable, leading to a transverse modulation which would 


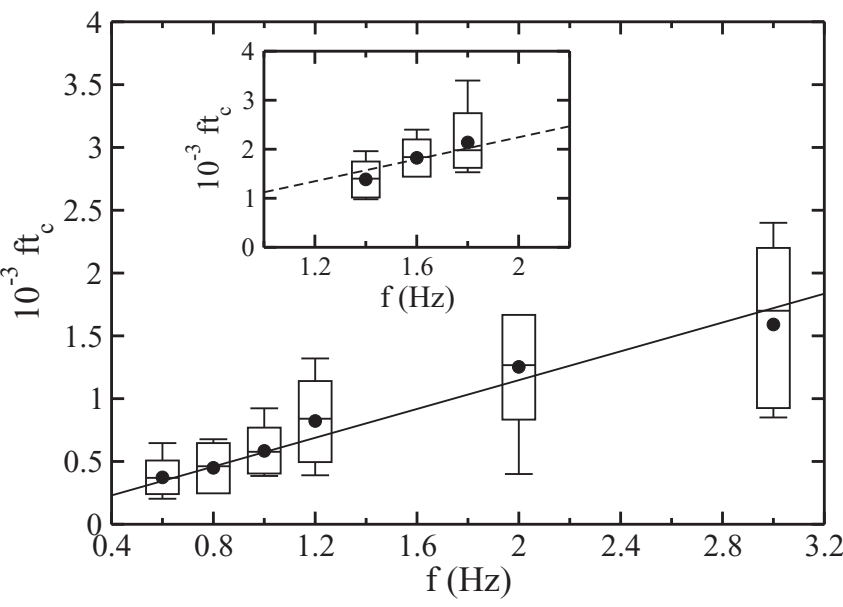

FIG. 4. Nondimensional threshold time $\left(f t_{c}\right)$ as a function of applied frequency. Box plots merge data for different strengths of the applied field. For each frequency, the mean nondimensional threshold time is represented by a filled circle. The lines are fits to a proportional law (solid corresponds to $t_{c}=574 \mathrm{~s}$ and dashed to $t_{c}=1122 \mathrm{~s}$ ). Inset corresponds to experiments performed with more hydrophilic substrates.

be responsible for the cluster formation. These effects are sketched in Fig. 6.

Initially, when the electric field is turned on, the particles get accumulated like a band near the contact line (Figs. 2(b) and 2(c)). At later stages these particles initiate the formation of clusters and they evolve. Similarly, as in the case of the response of particles, the clusters follow the force at lower order of applied frequency (Fig. 3, from 0.6 to $1.8 \mathrm{~Hz}$ ) by moving up and down as a whole (perpendicularly to the applied field). Thus, the clusters can follow the variations in the contact angle, as it is observed in the experiments, but contrarily, the measured frequencies of the clusters are higher for high applied

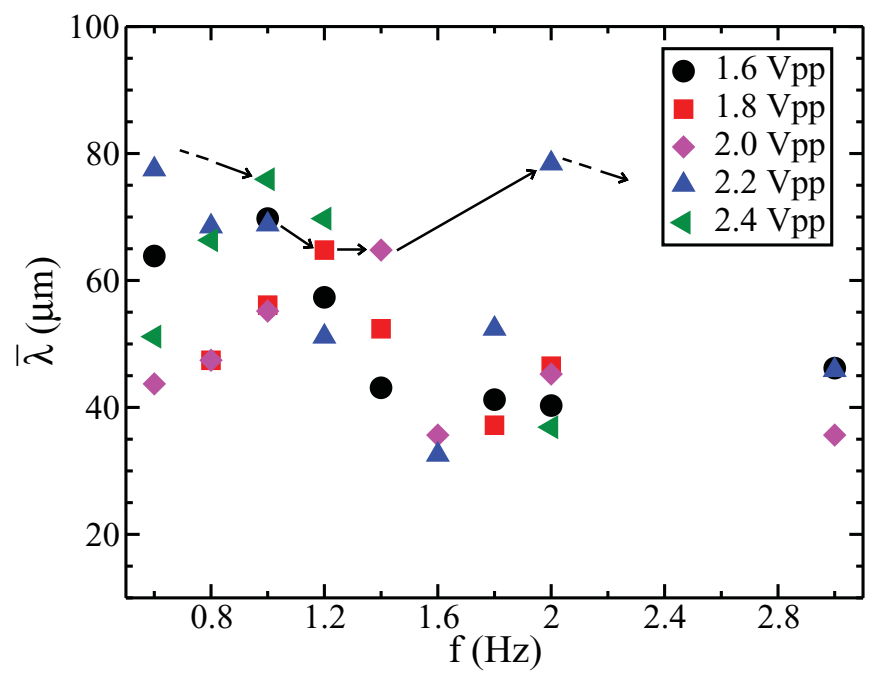

FIG. 5. (Color online) Characteristic length $(\bar{\lambda})$ at the threshold time $\left(t_{c}\right)$ as a function of applied frequency. The arrows outline the sequence of the maxima occurring while increasing the strength of the field. The dashed line arrows correspond to the 2.2-2.4 Vpp transition (see text).
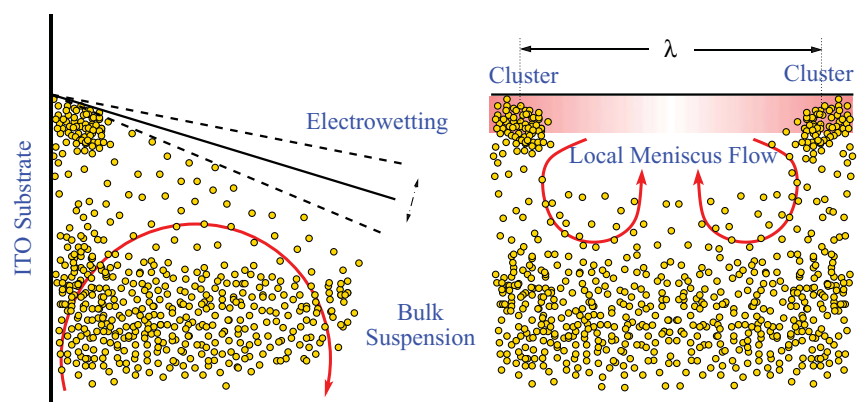

FIG. 6. (Color online) Microscopic sketch of the region near the contact line. Left: lateral view. Effect due to electrowetting has been enhanced for better view. Right: front view. Local flows are indicated by red (curved) arrows, and the corresponding transverse modulation in the contact angle is shown by the color (shade) gradient near the contact line.

frequencies. For these frequencies, the rapid variations in the contact angle make these clusters unable to follow and they do not move, due to their inertia. However, the particles rearrange inside the clusters. This could lead to high frequency variations in the measured light intensity without global movement of the clusters (see clip B in [24]).

The long temporal scale behavior of the system can be characterized by measuring the time at which the clusters evolve significantly. The proportional increase in the nondimensional time (Fig. 4) indicates that this global evolution of the system (time required for the clusters to age or dissociate) is weakly dependent on the forcing condition. The measured higher nondimensional time in Fig. 4 (inset) is due to the character of the substrate (less hydrophobic), and the aging is due to a global scale flow which disassembles the clusters (see clip A in [24]).

An instability of the flows (induced by the ac electrowetting) gives rise to transverse local flows and to a modulation of the varying contact angle (Fig. 6, right). These local flows are advection-like closed flows. We define the recurrence time as the time taken by a volume element to complete a cycle. The

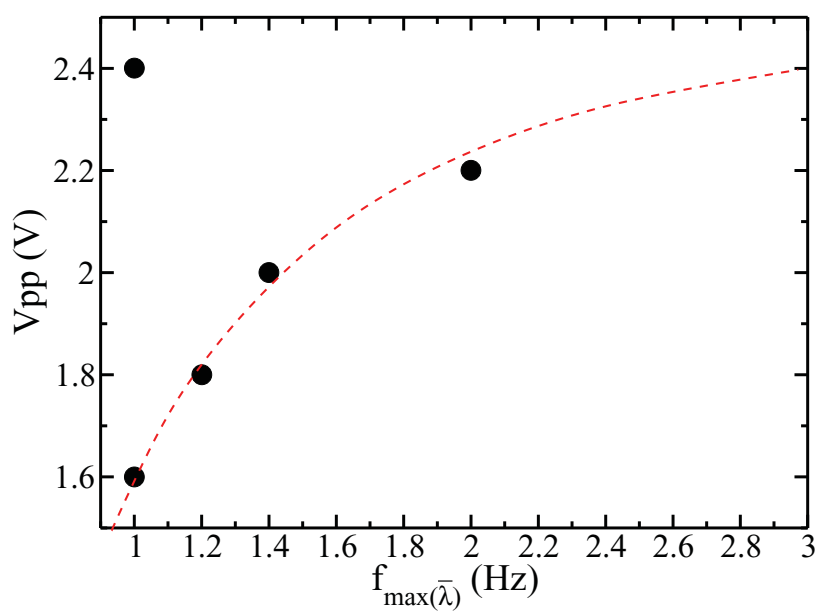

FIG. 7. (Color online) Correlation between applied amplitude and formation of clusters for a relative maximum in characteristic length $\bar{\lambda}$. The dashed line is a guide to the eye. 
characteristic length of these closed flows corresponds to the mean distance between clusters $(\bar{\lambda})$. The characteristic length changes as we increase the applied frequency (see arrows in Fig. 5). For a certain frequency, a relative maximum in the characteristic length is observed. This frequency depends on the recurrence time and thus on the strength of the local flows. To have this condition (relative maximum in characteristic length) for increasing applied frequencies, the amplitude has to be increased (Fig. 7). The recurrence time is limited in order to have a regular flow. Consequently, for a high applied amplitude (e.g., $2.4 \mathrm{Vpp}$ ) the relative maximum in characteristic length is obtained at a lower applied frequency that corresponds to a multiple of the expected recurrence time (see dashed line arrows in Fig. 5, and Fig. 7). This may indicate that the instability is parametric [27].

In conclusion, the applied alternating field generates flows near the contact line and clusters are formed. The clusters are separated by a well-defined characteristic length $(\bar{\lambda})$. The clusters are located in the meniscus and they survive in our experimental conditions between 5 and $15 \mathrm{~min}$. The response of the contact angle depends on the applied frequencies and it is measured through the frequency response of the clusters. Also, the applied field controls the flows in the meniscus (through variation of the contact angle). These results will be complemented in further work with the characterization of the instability (thresholds and type of bifurcation) and of the phase space. It is crucial to understand the underlying fluid dynamics in order to control the periodicity of the cluster array, which is very relevant in applications where colloidal multiscale structures are needed. For this, both new experiments and models are required. Our experimental setup and conditions allow construction of this kind of colloidal deposit at low cost and in a straightforward manner. Although we have determined that electroosmosis [28,29] does not play an important role in this experiment, more research is needed to address this issue quantitatively.

This work was partly supported by the Spanish Government, Contract No. FIS2008-01126, and by the Gobierno de Navarra (Departamento de Educación). M.P. and M.G. acknowledge financial support from the "Asociación de Amigos de la Universidad de Navarra."
[1] M. Trau, D. A. Saville, and I. A. Aksay, Science 272, 706 (1996).

[2] C. Arcos, K. Kumar, W. González-Viñas, R. Sirera, K. M. Poduska, and A. Yethiraj, Phys. Rev. E 77, 050402(R) (2008).

[3] A. Yethiraj, A. Wouterse, B. Groh, and A. van Blaaderen, Phys. Rev. Lett. 92, 058301 (2004).

[4] T. Gong, D. T. Wu, and D. W. M. Marr, Langmuir 19, 5967 (2003).

[5] Y. H-Tao, W. Ming, G. Y-Xian, and Y. Ping, Chinese Phys. B 18, 2389 (2009).

[6] A. Yethiraj, Soft Matter 3, 1099 (2007).

[7] M. Giuliani, M. Pichumani, and W. González-Viñas, Eur. Phys. J. Special Topics 192, 121 (2011).

[8] M. Cross and P. C. Hohenberg, Rev. Mod. Phys. 65, 851 (1993).

[9] D. Wirtz and M. Fermigier, Phys. Rev. Lett. 72, 2294 (1994).

[10] M. Giuliani, W. González-Viñas, K. Poduska, and A. Yethiraj, J. Phys. Chem. Lett. 1, 1481 (2010).

[11] K. Q. Zhang and X. Y. Liu, J. Chem. Phys. 130, 184901 (2009).

[12] W. D. Ristenpart, P. Jiang, M. A. Slowik, C. Punckt, D. A. Saville, and I. A. Aksay, Langmuir 24, 12172 (2008).

[13] A. S. Negi, K. Sengupta, and A. K. Sood, Langmuir 21, 11623 (2005).

[14] J. R. de Bruyn, Phys. Rev. A 46, R4500 (1992).

[15] M. P. Brenner, Phys. Rev. E 47, 4597 (1993).

[16] J. A. Diez and L. Kondic, Phys. Rev. Lett. 86, 632 (2001).
[17] Y. Hu, J. L. Glass, A. E. Griffith, and S. Fraden, J. Chem. Phys. 100, 4674 (1994).

[18] E. Schäffer, T. Thurn-Albrecht, T. P. Russell, and U. Steiner, Europhys. Lett. 53, 518 (2001)

[19] K. H. Kang, Langmuir 18, 10318 (2002).

[20] F. Mugele, A. Klingner, J. Buehrle, D. Steinhauser, and S. Herminghaus, J. Phys. Condens. Matter 17, S559 (2005).

[21] J. Yamanaka, H. Matsouka, H. Kitano, and N. Ise, J. Colloid Interface Sci. 134, 92 (1990).

[22] J. Yamanaka, H. Matsouka, H. Kitano, N. Ise, T. Yamaguchi, S. Saeki, and M. Tsubokawa, Langmuir 7, 1928 (1991).

[23] B. Ashok and M. Muthukumar, J. Phys. Chem. B 113, 5736 (2009).

[24] See supplemental material at [http://link.aps.org/supplemental/ 10.1103/PhysRevE.83.047301] for a movie which corresponds to Fig. 2 (clip A) and for a movie where the behavior of clusters at high frequency $(3 \mathrm{~Hz})$ can be seen (clip B).

[25] S. H. Ko, H. Lee, and K. H. Kang, Langmuir 24, 1094 (2008).

[26] H. Lee, S. Yun, S. H. Ko, and K. H. Kang, Biomicrofluidics 3, 044113 (2009).

[27] F. Poulin, G. Flierl, and J. Pedlosky, J. Fluid Mech. 481, 329 (2003).

[28] M. Trau, D. Saville, and I. Aksay, Langmuir 13, 6375 (1997).

[29] Y. Solomentsev, M. Böhmer, and J. Anderson, Langmuir 13, 6058 (1997). 